the light chain $\mathrm{V}$ region of myeloma MOPC 315 which produces lambda chains of subgroup ii. A curious feature of the sequence is that at three of four of the positions where it disagrees with the MOPC 315 sequence it corresponds to that of MOPC 104R, which produces lambda $\mathrm{i}$ light chains; in the fourth position it agrees with neither. C. Milstein (MRC Cambridge) gave voice to a general feeling that this pattern of substitutions is unlikely to be random. More sequence data should clarify the issue, but in the meantime the data are reminiscent of the amino acid sequences of mouse kappa V regions, reported by $M$. Weigert (Institute for Cancer Research, Philadelphia). Some residues in these chains seem to switch between closely related subgroups.

Whether more amino acid sequence data will help to clarify the question of the generation of antibody diversity is controversial. Amino acid sequences were presented at the meeting in support of an insertion theory (D. Capra, University of Texas) and of a somatic mutation theory (Weigert) but neither set of data could strictly eliminate alternative theories. It is now generally conceded that antibody diversity arises by a somatic mechanism acting on a limited number of germ-line genes (probably two or three copies for lambda chains and perhaps two or three copies for each of an unknown number of kappa chain subgroups) (Rabbitts \& Milstein Contemporary Topics in Molecular Immunology 6, 117; 1977; Tonegawa et al. Immun. Rev. 36, 29; 1977) and there is a school of thought that the solution to the generation of diversity will be found in cellular mechanisms rather than sequences. In the meantime, the rational position on GOD seems to be agnostic.

\section{Idiotypes and immunoregulation}

Sequence data may, on the other hand, help to clarify the structural and functional significance of idiotypes, which if Jerne is right may be the basis for intercellular recognition in a regulatory network. At what levels might Jerneian immunoregulation take place? Capra presented unequivocal evidence that the so-called 'anti-Ars' idiotype on mouse anti-arsonate antibodies is determined by specific residues in the hypervariable regions which also determine antigen-binding specificity. However, there is ample serological evidence that idiotype is not always associated with the antigen-binding site, and T. Feizi for instance (MRC Clinical Research Centre, Harrow) has found that crossreacting idiotypes on human antibodies against erythrocyte Ii antigens can also be found on antibodies which lack that binding specificity. In competitive bind-

\section{Unification confrontation}

Most physicists hope that the electromagnetic and weak interactions are unified and this hope has been strengthened over the past few years as the Weinberg-Salam unified model of the weak interactions has notched up success upon success. But a small cloud appeared on the horizon last year; and it has not gone away.

Take the Weinberg-Salam model, add some atomic physics calculations, and the result is a prediction of small, but measurable, parityviolating effects in atoms. Preliminary results from experiments aimed at detecting these effects were reported last year (Nature 264, 528; News and Views 264, 505; 1976) and were disturbing-the predicted effects were not seen.

These experimenters now have their final results (Phys. Rev. Lett. 39, 795; 39, 798; 1977) and have reached a sensitivity an order of magnitude better than the prediction. No parity-violating effects are seen and the threat to the unified theories must now be regarded as serious.

There is only one foreseeable escape route-perhaps the atomic physics calculations for the bismuth atoms used in these experiments could be in error. Experiments planned with hydrogen will resolve this final uncertainty.

ing assays, the idiotypes can be identified as a heavy chain framework determinant that seems to identify a sub-subgroup.

Subgroups of subgroups were a particular feature of Weigert's partial sequence data on 22 mouse kappa light chains, and raised the question of how variable a framework residue has to be before it becomes part of a hypervariable region. (Is GOD leaky? asked Milstein). Indeed Weigert and his colleagues have now found in mouse light chains a fourth distinct hypervariable region, like that reported earlier by Capra et al. in heavy chains. The fourth hypervariable region (like the second, at least in those antibodies whose crystal structure is known) is remote from the antigen-binding site.

F. Melchers (Basel Institute for Immunology) was enormously interested in these results, on the grounds that the fourth hypervariable region might provide the basis for recognition in Jerne's immune network. He has indirect evidence for idiotypic regulation of mouse antibodies to streptococcus A carbohydrate, which carry the A5A idiotype. Primary immunisation with either the streptococcal antigen or antibodies against the A5A idiotype stimulates the production of idiotypepositive antibodies $40 \%$ of which bind the antigen and $60 \%$ of which do not, regardless of the priming stimulus. Melchers interprets these resultswhich strictly apply only to lipopolysaccharide (LPS)-sensitive cells (one third of B cells) since LPS was used to expand the number of cells in vitro for counting - as evidence for lymphocyte triggering by idiotype rather than by antigen-binding site in these cells.

The structural significance of idiotype arose again in connection with the $T$ cell receptor-another crucial element in any immune network. The recent discovery that $\mathrm{T}$ and $\mathrm{B}$ cells can carry the same heavy chain idiotype and that anti-idiotype antibodies can trigger $\mathrm{T}$ cell help in vitro (see Cold Spring Harbor Symp. quant. Biol. 61, 1976 and News and Views 263, 10; 1976) has been interpreted to imply a $\mathrm{T}$ cell antigen receptor coded partly by genes in the pool coding for the $\mathrm{V}$ region of the heavy chain. However, $\mathrm{K}$. Rajewsky's group, as reported by T. Cramer and T. Imanishi (Cologne University), now finds that in SJL mice $\mathrm{T}$ cells but not $\mathrm{B}$ cells carry an idiotype associated with the heteroclitic binding of the hapten nitrophenol (NP). Genetic experiments suggest that this heavy chain idiotype is expressed only in cells which produce lambda light chains and its absence in SJL B cells is due to the very low production of lambda chains by that strain. This leaves a puzzle: what produces the conformational change that gives the idiotype on SJL T cells? So far, the evidence has been against any light chain gene contribution to the 'T cell receptor.'

The proper characterisation of the $\mathrm{T}$ cell receptor will really be possible only when one can be isolated in large quantities. This may be soon. G. Fathman and $H$. Hengartner (Basel Institute of Immunology) reported a technique for producing 3-month cultures of $T$ cells with specific reactivity in a mixed lymphocyte culture-a potential source of receptors for histocompatibility antigens.

\section{'Hybridomas'}

The final session of the meeting amounted to a progress report on the developing range of hybridomas. Since its beginning with immortal clones producing antibodies against sheep red blood cells, the hybridoma army has recruited clones producing antibodies against haptens TNP, NIP and NP; soluble proteins peroxidase, lysozyme, and chick gamma-globulin; and influenza virus surface antigens, rat 